\section{DMD's leaky faucet}

\section{By Lauren Martz, Staff Writer}

A team at Columbia University has found a new target for muscular dystrophy-a leaky RyR1 channel. Blocking the channel's calcium leak from the sarcoplasmic reticulum could help protect skeletal muscle from damage caused by Duchenne muscular dystrophy. ${ }^{1}$ Armgo Pharma Inc. has already licensed the patents arising from the research.

Duchenne muscular dystrophy (DMD) is an X-linked genetic disorder that affects about 1 in 3,500 males, according to NIH's National Center for Biotechnology Information. The disorder results in a loss of the protein dystrophin, which disrupts both the dystrophin-glycoprotein complex and muscular calcium homeostasis. The damage resulting from the loss of dystrophin can lead to early death, often due to cardiac or respiratory muscle damage. ${ }^{2}$

Standard care is high-dose corticosteroids, which delay progression but come with a host of side effects and have no disease-modifying effects. ${ }^{3}$

In a paper published in Nature Medicine, Andrew Marks and colleagues at Columbia University College of Physicians and Surgeons identified a new strategy to treat or prevent the symptoms of DMD. The team reported that S-nitrosylation of the ryanodine receptor 1 (RyR1) channel-which occurs in the $m d x$ mouse model of DMD and therefore could be occurring in human patients-depletes the stabilizing subunit calstabin-1 from the channel's sarcoplasmic-bound complex. The result is a calcium leak.

Levels of calstabin-1 were decreased in $m d x$ mice compared with those seen in littermate controls. The researchers showed that stabilizing the channel prevented the calcium ion leak. Mice treated with S107, a RyR1 calcium ion release channel stabilizer, had better grip strength and exercise than that seen using vehicle control.

S107 delivered by osmotic pump lowered serum concentrations of creatine kinase, a marker of muscle necrosis, and levels of calpains, which are activated by high cytoplasmic calcium ion levels. The compound also improved multiple histological hallmarks of dystrophy.

Administration of S107 through drinking water for 7-10 days improved force generation compared with that seen using controls in mice whose muscles underwent eccentric contraction, a lengthening of the muscle from an external force. Finally, inducing and elongating muscle contraction in $m d x$ muscle decreased the measure of force compared with that seen in wild-type controls.

\section{Stabilizing the stabilizer}

Although RyR1 is a new target, S107 is unlikely to be taken forward in development.

Marks told SciBX that S107 has a short half-life, which might make it unsuitable for clinical development. He said his group's next steps include selecting a compound with better pharmacokinetics for clinical trials. Marks, corresponding author on the paper, is chair of the Department of Physiology and Cellular Biology and founding director of the Helen and Clyde Wu Center for Molecular Cardiology at Columbia.

Jon Tinsley, senior director of therapeutic programs at Summit plc, agreed that a better calcium channel stabilizer is needed. "The compound in the paper had to be delivered via osmotic pump or drinking water. This might mean that it must be continuously pumped in and improvements to the compound are necessary," he said. "Although DMD can kill patients in about 20 years and the demand for effective treatments is extremely high, some sort of tablet or injection is still highly preferred."

Summit's SMT C1100, a small molecule that upregulates utrophin, is in preclinical testing to treat DMD. The compound is partnered with BioMarin Pharmaceuticals Inc.

The compound class to which S107 belongs-rycals-also could be an issue. According to Ellen Welch, associate director of biology at PTC Therapeutics Inc., the class has been used on cardiac targets and has shown effects on other channels such as the potassium channel. Therefore, using this class of compounds could cause cardiovascular off-target effects when used in the DMD indication.

PTC's ataluren is in pivotal Phase IIb trials to treat DMD patients with a nonsense mutation in the gene encoding dystrophin. About 15\% of DMD patients have nonsense mutations. The compound, a small molecule that facilitates the complete translation of proteins containing nonsense mutations, is partnered with Genzyme Corp.

Robyn Murphy of La Trobe University agreed that S107 "is likely to also have effects on cardiac muscle. It will therefore be prudent to test the efficacy of $\mathrm{S} 107$ on healthy animals as well as examining cardiac sarcoplasmic reticulum function in models of DMD." Murphy is a National Health and Medical Research Council postdoctoral research fellow in the Department of Zoology at La Trobe.

\section{Muscle models}

In addition to improving the drug-like properties of S107, other researchers wanted to see the findings validated in a model that more accurately reflects human pathology. 


\section{TARGETS \& MECHANISMS}

"The $m d x$ mouse differs in its phenotype in several regards from DMD patients," said Oliver Friedrich, senior research fellow at the school of biomedical sciences of the University of Queensland. "Unlike humans, limb muscles in $m d x$ mice show a very mild pathology."

Murphy agreed that the $m d x$ mouse might be too mild of a model. Although the animal's muscle atrophy is "typical of this type of dystrophy in the first weeks of life, it is able to recover somewhat, and muscle regeneration occurs," she noted.

Thus, Murphy said, "it is not a great model for DMD, although it is able to provide valuable preliminary data to formulate hypotheses that can be further tested in better models for DMD, such as the canine X-linked muscular dystrophy model or the dystrophin and utrophin double knockout mouse."

Friedrich agreed that "a possible next step should be to also test the drug in dystrophic golden retriever dogs, an animal model that is closer to human pathology."

Tinsley thinks the work in the mouse could have been more extensive. "The researchers performed one experiment where they took the muscle out, stretched and contracted it using eccentric contraction, and showed that with the compound, the muscle was more resilient to damage," he said. "But they only electrochemically contracted the muscle once. Most DMD researchers would know to do that maybe five more times to determine whether it could withstand the effects of the physical damage that would occur."

Tinsley added, "Longer dosing studies would also be necessary, and it would be good to look more specifically at the effects on the muscle fibers than just the properties such as grip strength.”

\section{Trouble with RyR1}

If RyR1 stabilization does prove to be sufficient to protect muscles in $\mathrm{DMD}$, specifically targeting the channel could provide additional hurdles to drug development.

"There are other tissues that express RyR1 proteins, such as the hippocampus, so the development of a skeletal muscle-specific inhibitor might be necessary," Welch said.

Friedrich also noted that RyR1 has multiple regulatory binding sites and that "there are many disorders related to RyR1 dysfunction." As a result, he said, a molecule that hits the channel "might alter binding of other constituents needed for normal fine-tuning of RyR1 function."

To test for such effects, Friedrich suggested "incorporating isolated RyR1 into bilayer membranes to study their biophysical properties" in the presence of a therapeutic candidate.

Even if a molecule can be developed that affects RyR1 only in cells or tissues relevant to DMD, he noted "that absence of dystrophin seems to affect multiple calcium targets. For example, different ion channels permitting increased calcium entry into the cell have been shown to be affected, like calcium leak channels, mechanosensitive channels, voltage-sensitive channels and store-operated channels."

Despite the challenges, Welch thinks the larger goal of blocking calcium leak should be pursued. "Preventing the calcium leak would go a long way to maintaining the muscle cell population," she said.

Friedrich agreed. "It seems that preventing the calcium leak effectively prevents downstream mechanisms that are triggered by calcium overload that otherwise occur through this leak, like inflammation, necrosis and muscular degeneration/regeneration cycles. Also, by reducing the leak there is more calcium available in the stores for normal muscle activation in dystrophic muscle."

"Anything that can prolong the life of the muscle fibers could play an important role in DMD treatment, because we call DMD a disease with unmet medical need that is $100 \%$ lethal," said Tinsley.

Although the most sought-after therapeutic option remains fixing the root cause of the disease-loss of dystrophin-Friedrich said that preventing the calcium leak through RyR1 could help patients who might not benefit if a curative therapy is found.

"With age, muscles become more and more deteriorated and the morphology probably irreversibly affected," he said. "In an adult patient, gene therapy might come too late to correct for already dominant muscle wasting, necrosis and cardiomyopathy. Maybe supportive drug therapy can be of benefit at this stage."

Friedrich suggested that the next steps could include an agerelated study to determine whether an RyR1 stabilizer can reverse the disease process.

Marks told SciBX that the findings covered in the Nature Medicine paper have been patented by Columbia University in the U.S. and other countries. The patents have been exclusively licensed by Armgo Pharma and are available for sublicensing in the U.S. and Japan.

Carolyn Paradise, president and COO of Armgo, said the company is interested in pursuing both the heart and skeletal muscle applications of the research from Marks' lab. The company's therapeutic program for muscle disorders is in lead optimization, whereas its most advanced compound, also targeting RyR1, is in Phase I testing to treat chronic heart failure.

Martz, L. SciBX 2(8); doi:10.1038/scibx.2009.303

Published online Feb. 26, 2009

\section{REFERENCES}

1. Bellinger, A. et al. Nat. Med.; published online Feb. 8, 2009; doi:10.1038/nm.1916

Contact: Andrew R. Marks, Columbia University College of Physicians and Surgeons, New York, N.Y. e-mail: arm42@columbia.edu

2. Hoffman, E. et al. Cell 51, 919-928 (1987)

3. Fisher, l. et al. FASEB J. 19, 834-856 (2005)

\section{COMPANIES AND INSTITUTIONS MENTIONED}

Armgo Pharma Inc., New York, N.Y.

BioMarin Pharmaceuticals Inc. (NASDAQ:BMRN), Novato, Calif. Columbia University, New York, N.Y.

Genzyme Corp. (NASDAQ:GENZ), Cambridge, Mass.

La Trobe University, Victoria, Australia

National Institutes of Health, Bethesda, Md.

PTC Therapeutics Inc., South Plainfield, N.J.

Summit plc (LSE:SUMM), Abingdon, U.K.

University of Queensland, Brisbane, Queensland, Australia 